\title{
Clinical, pathologico-anatomic and mycological studies of aspergillosis in ostrich (Struthio camelus)
}

\author{
Riaz Hussain 1*, Fazal Mahmood², Ghulam Murtaza ${ }^{3}$, Muhammad Ismail Abbas ${ }^{4}$, Jawad Munawar Warraich ${ }^{4}$, \\ Rabia Akram $^{5}$ \\ ${ }^{1}$ Department of Pathology, Faculty of Veterinary and Animal Sciences, The Islamia University of Bahawalpur 63100, \\ Pakistan \\ ${ }^{2}$ Department of Pathology, Faculty of Veterinary Science, University of Agriculture, Faisalabad 38040, Pakistan \\ ${ }^{3}$ Livestock Production Research Institute, Bahadurnagar, Okara, Pakistan \\ ${ }^{4}$ Poultry Research Institute, Rawalpindi, Pakistan \\ ${ }^{5}$ Institute of Pure and Applied Biology, Zoology Division, Bhauddin Zakariya University, Multan, Pakistan
}

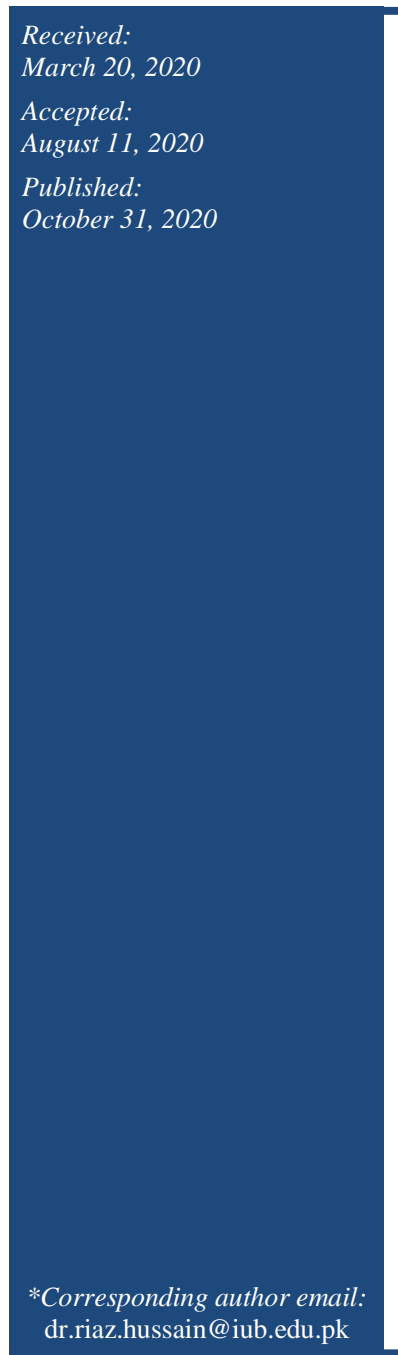

\begin{abstract}
Aspergillosis is known as the most commonly occurring mycotic infection in variety of avian species causing significant economic losses in terms of poor growth, decrease in overall production and/or mortality in different species of birds including ostrich throughout the world due to aspergillosis. This study describes clinical, histopathological and mycological findings in an adult ostrich. An adult ostrich with history of simultaneous occurrence of blocked bladder associated with penile protrusion, proventriculus impaction and testicular ailments died of aspergillosis infection. Different clinical signs such as anorexia, dyspnea and coughing were observed prior to death. Necropsy findings showed significant enlargement of urinary bladder impacted with yellow color and amorphous material. Spherical grayish white raised areas of caseous necrotic foci of variable diameter were sparsely spread over the air sacs. Proventriculus was full of gravel; iron bars corn cobs and plastic bottles. Multiple solitary well circumscribed nodules with hard consistency, hanging with fibrous threads in air sacs were packed with yellow cheesy material. Grossly the testes were smaller in size and hard in consistency. Histologically, the in testes atrophy seminerous tubules, increased connective tissue proliferation and chronic inflammatory cell infiltration was observed. Moreover, the seminerous tubules were lined by one to two layers of germinal epithelial cells exhibiting degenerative and necrotic changes and reduced process of spermatogenesis. Some tubules showed obliterated lumen and multinucleated giant cells with engulfed necrotic cells were also observed. Results on mycological examination, staining with lactophenol, Giemsa's and florescent stain revealed presence of prominent fungal hyphae and sporangium. Histologically, the lungs tissue revealed multiple areas of caseous necrosis. The current study describes novel finding of blocked bladder and fungus infection in an ostrich that died of aspergillosis.
\end{abstract}

Keywords: Ostrich, Aspergillosis, Bladder, Testes, Histopathology

\section{How to cite this:}

Hussain R, Mahmood F, Murtaza G, Abbas MI, Warraich JM and Akram R, 2020. Clinical, pathologico-anatomic and mycological studies of aspergillosis in ostrich (Struthio camelus). Asian J. Agric. Biol. 8(4): 386-391. DOI: https://doi.org/10.35495/ajab.2020.03.145

This is an Open Access article distributed under the terms of the Creative Commons Attribution 3.0 License. (https://creativecommons.org/licenses/by/3.0), which permits unrestricted use, distribution, and reproduction in any medium, provided the original work is properly cited. 


\section{Introduction}

Ostrich are large birds with high economic potential and can easily adapt to various climatic conditions in tropical and sub-tropical parts of the world. The increasing trend towards ostrich breeding has been paid in different parts of the world to fulfill the requirements of meat and eggs (Khosravi et al., 2008). Among different species of birds, ostriches (Struthio camehts) are the largest living bird in the world. These birds can survive even in very harsh environment and are considered as one of the strongest birds on the earth. Ostriches are very resistant to many infections however, they may suffer with respiratory distress as a result of frequent growth of fungus particularly aspergillosis (Beernaert et al., 2010). Aspergillosis is a predominantly disease of respiratory tract caused by non-contagious and ubiquitous fungal species. Although the disease causes mainly respiratory ailments but can cause mortality in free-living and captive birds involving multiple organs (Khosravi et al., 2008; Cacciuttolo et al., 2009; Beernaert et al., 2010). As a result of aspergillus growth within the air sacs the birds became more susceptible to respiratory infections which can be acute or chronic in nature such as pneumonia. Clinically affected ostriches due to aspergillosis infection revealed anorexia, cyanosis of mucous membranes and even acute mortality. Chronic aspergillosis is much more common (Yalcin et al., 2018) and is characterized by development of fungal plaques within the air sac and formation of local multiple well circumscribed granulomas in lungs (Jung et al., 2009). Migration of spores either through blood circulation or lymphatic system may lodge to other organs particularly kidney, liver and proventriculus (Akkot et al., 2009; Cray et al., 2009b). Aspergillosis in birds caused by fungi of the genus Aspergillus in different avian species such as ostrich, pigeon, chicken, penguin, canary, turkey and crow is a well-known respiratory infection (Sana et al., 2019). Aspergillosis is a devastating infection and is characterized by severe pulmonary edema and zvgomycosis in ostriches and different other avian species including turkeys, chickens, quail, pigeons and penguins (Reissig et al., 2002; Khosravi et al., 2008). Extension of fungus growth to deeper areas of the lungs is usually evident with harsh lung sounds during the auscultation of thoracic cavity of the ostrich (Cooper, 2005). Previously, it has been reported that aspergillosis causes 5-20\% mortality in young ostriches, while 5\% high mortality in adult ostrich as compared to young birds (Femenia et al., 2007; Akkot et al., 2009): Studies have observed increased prevalence of aspergillosis in ostriches in different parts of the world particularly Israel and Iran (Shahrzad et al., 2014), and Turkey (Asan, 2004).Various pathological lesions in different tissues of birds died of aspergillosis show characteristic features of disease. Previously various characteristic features of aspergollosis such as presence of caseous nodules in lungs, air sacs, liver, kidneys, spleen, bursa and glandular stomach have been reported by different researchers (Gulbahar et al., 2000; Atasever and Gümüșsoy, 2004; Akkot et al., 2009). Current scientific knowledge of diseases of ratite birds (ostriches, emus and rheas) is incomplete, fragmented and in most cases superficial or limited to anecdotal reports. In Pakistan, many people are involved in ostrich farming on commercial scale even without government support. This report presents a novel finding of blocked bladder and fungus infection in an ostrich that died of aspergillosis in the district of Faisalabad.

\section{Material and Methods}

\section{History and pathological investigation}

A 5- year sexually mature and adult male ostrich died at a small zoo with the history of respiratory distress, anorexia, trembling, oligouria, straining at the time of urination, pain and protrusion of the penis from the vent (Fig.1).

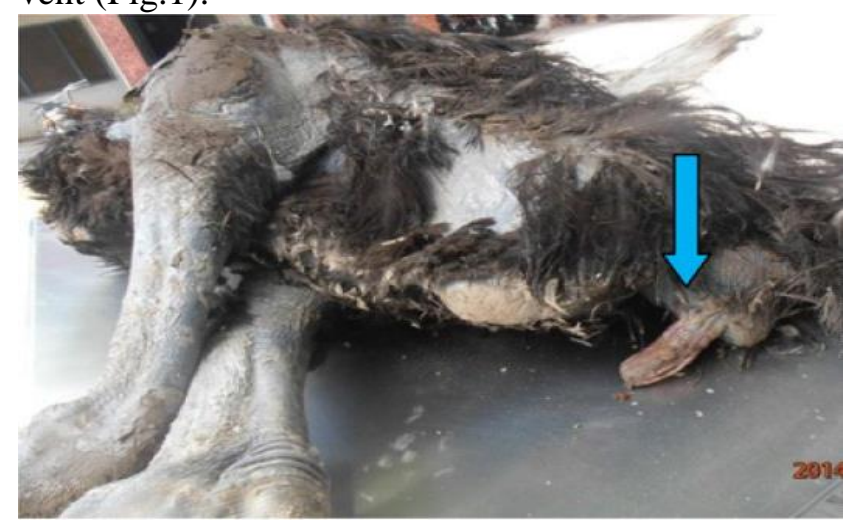

Figure-1. Photograph of male ostrich showing protrusion of the penis from the vent.

The necropsy of ostrich was conducted and the samples of the lungs, trachea, testes, pharynx and thoracic air sacs were fixed in $10 \%$ neutral buffered formalin, processed through paraffin method. The 4$5 \mathrm{pm}$ thick sections were stained with haematoxylin and eosin (Hussain et al., 2019). 


\section{Mycological examinations}

For mycological examinations tracheal and pharyngeal swabs and tissue samples from lungs and air sacs were collected at the time of necropsy. All these tissue samples were collected in sterile glass petri plates. The samples were inoculated on Sabouraud dextrose agar supplemented with chloramphenicol $(0.05 \mathrm{mg} / \mathrm{mL})$. All the inoculated petriplates were then incubated at $37^{\circ} \mathrm{C}$ for $3-5$ days under aerobic conditions. Finally, the morphological features of the culture were examined through light microscopy after Giemsa's staining, lactophenol and fluorescent dyes (Khosravi et al., 2008).

\section{Results}

Various clinical signs such as anorexia, dyspnea and coughing were observed in ostrich infected with aspergilosis before death. At necropsy different prominent lesions were observed in abdominal as well as pelvic cavity of the ostrich. The urinary bladder was extensively distended and filled with yellow color turbid urine along with large quantity of yellow color amorphous crystals (Fig. 2), impacting the external opening of the urinary bladder.

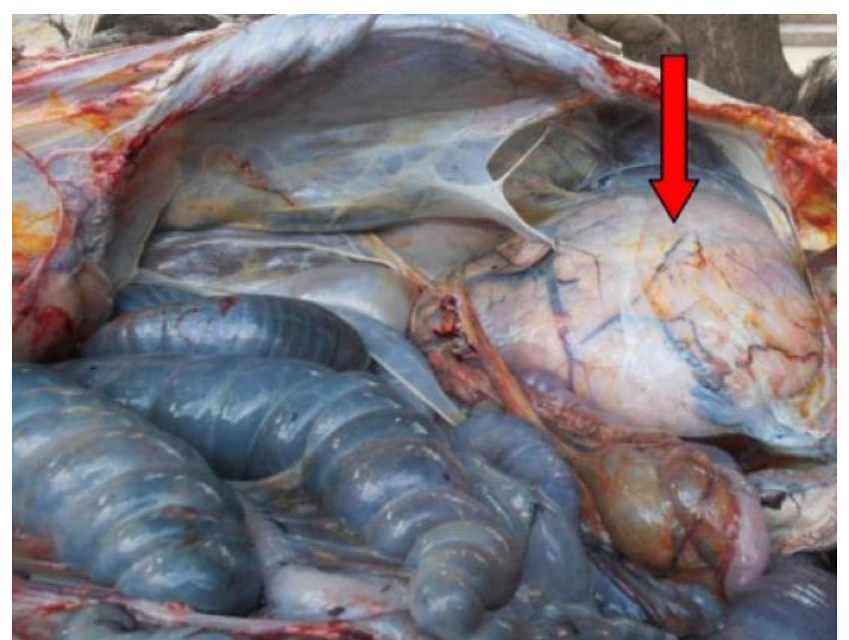

Figure-2. Photograph of male ostrich showing distention of urinary bladder filled with yellow color turbid urine.

Kidneys were swollen and paler in color. Proventriculus was distended and impacted with many corn cobs, gravel and plastic bottles. The visceral and parietal surfaces of thoracic and abdominal air sacs were embedded with solitary hanged nodules with fibrous thread (Fig. 3). It was hard in consistency, incised surface showed the packing of yellow cheesy material, however; numerous greenish to whitish velvety surfaced foci of variable sizes were present over the abdominal air sacs. Although lungs were normal in color and consistency but pleura was firmly attached to the costal surfaces. At postmortem levels, different lesions like thick air sacs containing exudates, white multiple nodules in spleen and liver tissues were observed. Microscopic examination of lung tissue revealed micro nodules with caseous center and fungal granuloma, exhibiting numerous hyphae, mycelia in the middle of granuloma, brim of which was punctuated with chronic inflammatory cells including lymphocytes, plasma cells and fibroblasts.

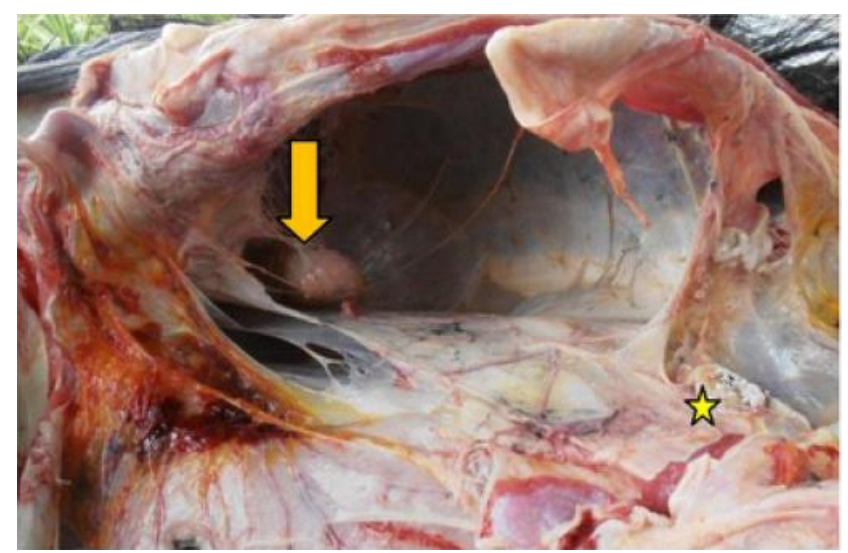

Figure-3. Photograph of male ostrich showing parietal surfaces of thoracic and abdominal air sacs embedded with solitary hanged nodules and fibrous threads.

Moreover, the kidneys showed congestion and sporadic necrosis of tubular epithelial cells. Most of the tubular lumens were impacted with oxalate crystals. Mild to moderate degenerative changes in the liver tissue with cytoplasmic vacuolation of the hepatocytes were also observed. Grossly the testes were smaller in size and hard in consistency. Grossly the testes were smaller in size and hard in consistency. Histologically, the testes showed various alterations including increased connective tissue proliferation in interstistium, chronic inflammatory cell infiltration, severe testicular tubular degeneration, seminiferous tubules were lined by one to two layers of spermatogonia without secondary spermatocytes and spermatozoa. The germinal tubular epithelial cells were exhibited degenerative and necrotic changes and the lumen of seminiferous tubules was obliterated containing mixture of dead cells. Multinucleated giant cells with engulfed necrotic cells were also observed (Fig. 4). Microscopic examination 
of culture with Giemsa's staining, lactophenol and fluorescent dyes revealed presence of fungal hyphae and sporangium (Fig. 5).

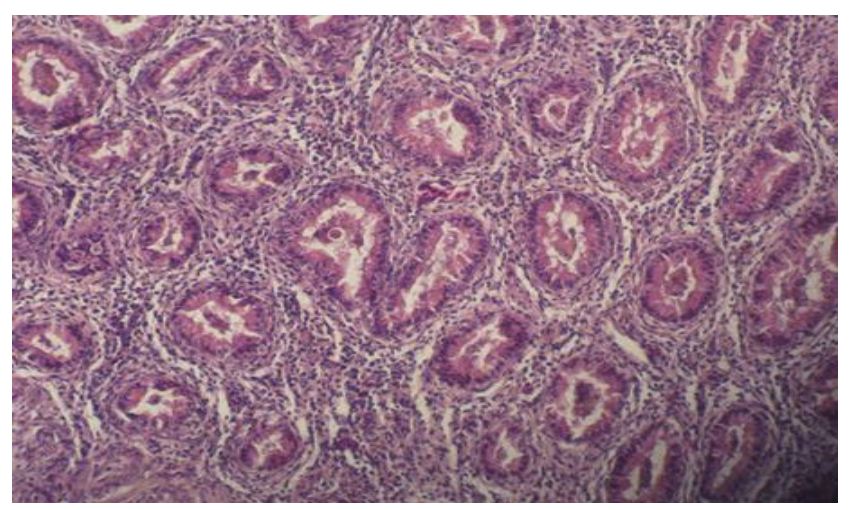

Figure-4. Photomicrograph of testes of male ostrich showing increased connective tissue proliferation in interstistium along with chronic inflammatory cell infiltration (H\&E stain; X-100)

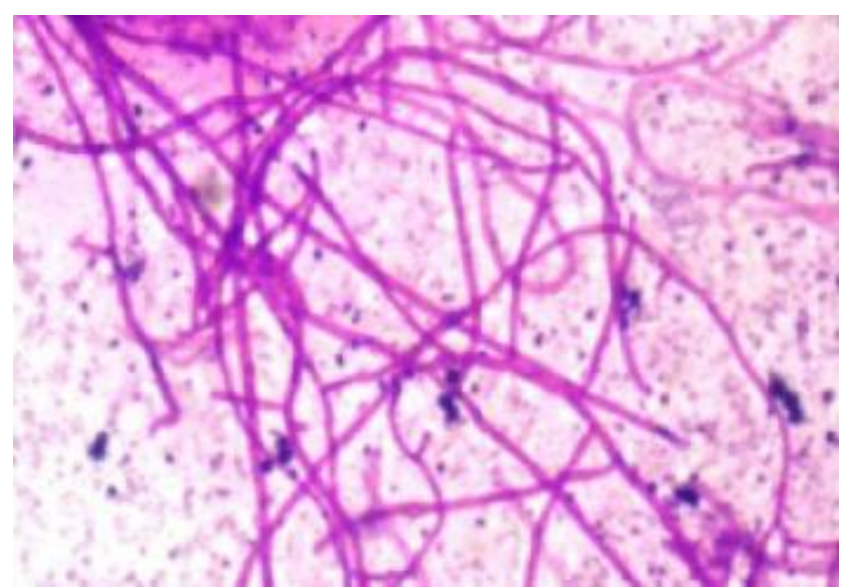

Figure-5. Photomicrograph showing presence of fungal hyphae and sporangium

\section{Discussion}

Different clinical signs such as anorexia, dyspnea and coughing were observed in infected ostrich. Clinical and behavioral manifestations in exposed avian species depend upon various factors such as infective dose, spore distribution, the immune response of the infected host and pre-existing diseases (Khosravi et al., 2008). Infections due to aspergillosis in birds are chronic and acute in nature and mainly results due to the inhalation of number of spores leading to immune suppression (Khosravi et al., 2008). Although the aspergillosis is a predominantly infection of respiratory tract however, it can infect different tissues and results malformation of the nostrils, exudative rhinitis, periorbital swelling, adhered and swollen eyelids, mycotic keratitis and blepharospasm (Hoppes et al., 2000). Ostriches (Struthio camelus) and different other avian species including kiwi, rhea, emu and cassowary are considered as economically important. These avian species have unique and organized reproductive system with complete separation of urine and feces (Sales, 2006). Generally these birds do not have the urinary bladder which is very unique feature. The urinary bladder in these birds corresponds to the ventral sac of coprodeum. These birds may exhibit morphological adaptation and may not be able to lower their weight through urination and defecation. Present findings of impaction of urinary bladder with heavy amorphous crystals of calcium may suggest that as compare to other avian species, coprodeum in ostrich plays a little role in post urethral modification of urine (Bezuidenhout, 1999). Further clarification of the post-renal modification of urine in the species must await meticulous comparison of the osmolality of ureteral and voided urine in the dehydrated state and precise measurements of the transport properties of epithelium. The role of mucin in establishing a thick unstirred layer should be quantified. In male ostrich the length of colon is about eleven meter and it works as a large cavity of fermentation to produce short chain fatty acids (Sales, 2006). The ostrich has a feature of survival without water drinking due to the subsequent absorption of these solutes to colonic water absorption (Viljoen et al., 2005). Furthermore, in ostrich urine retrograde movement is not found because of the existence of a sphincter between terminal colon and coprodeum (Bezuidenhout, 1999). It seems that the function of the coprodeum is like separate chamber to store the urine like urinary bladder, thus fulfilling one of the predicted criteria for the "mammalian" model. In this species there is renal concentrating feature resulting in the storage of urine (Dawson et al., 1985). This is actually a compensatory mechanism of dehydration in the arid and semi-arid zones where the ostrich is exposed to the dehydrating conditions. In Pakistan, environmental temperature during the summer month is significantly high that would be responsible for enhanced panting and water loss in these ostriches which predisposes the excessive accumulation of urates within the kidney parenchyma as well as in the urinary bladder and marked dilation of the urinary bladder. Moreover, it is considered that in urinary tract the secreted mucus is responsible to produce a thick unstirred layer at the surface of epithelium (Sales, 2006). 
In present study severe clinical signs in ostrich might be related to formation of calculi within the urinary bladder thus causing death. Previously, the urinary bladder distension in ostrich with penile protrusion has never been reported. However, urinary crenation has been reported in various other avian species due to accumulation of uric acid crystals. The possible reasons for the deposition of urates could be due to presence of organic matter in urine, derangement in the calcium metabolism, excessive ingestion in dietary minerals including heavily contaminated di-calcium phophate in feed and excessive use of non-steroidal anti-inflammatory drugs. It is reported that aspergillosis appears to be more significant in containment situations where predisposing factors such as infected hatcheries, contamination of air, soil, feed, stress and immunosuppression are involved (Khosravi et al., 2008). In young ostriches, aspergillosis may induce acute attacks and death with few days while in adult ostriches the infections mainly results in chronic form and are characterized by granulomatous lesions in the lungs and respiratory sacs. Similar to earlier reports, the infected ostrich in present study revealed the chronic fungal granuloma including lesions in thoracic air sacs (Khosravi et al., 2008). Multiple grayish white fungal niches within the lower air sacs and micro-biofilms in pulmonary parenchyma were the characteristic features and have been reported previously (Nardoni et al., 2006; Khosravi et al., 2008; Shahrzad et al., 2014). These fungal niches elicited a chronic inflammatory response in the lungs (Nardoni et al., 2006; Khosravi et al., 2008). Various pathological lesions like thick air sacs containing exudates, white multiple nodules in spleen and liver tissues in ostrich due to aspergillosis have also been observed (Khosravi et al., 2008; Shahrzad et al., 2014). Moreover, various lesions due to mycological infection in lungs, air sacs, liver, kidneys, spleen, bursa and glandular stomach have been observed (Atasever and Gümüșsoy, 2004; Akkot et al., 2009). Microscopic lesion in liver tissues like cytoplasmic vacuolation of the hepatocytes might be due to increased levels of oxido-nitrosative stress and up regulation of renal Caspase-3, KIM-1, TGF- $\beta$, and TNF- $\alpha$ mRNA expression in hepatic tissues (Adil et al., 2015). Histologically, the testes were prominently atrophied. Testes showed increased connective tissue proliferation in interstitium, chronic inflammatory cell infiltration, severe tubular degeneration, arrest of process of spermatogenesis and necrosis of primary and secondary spermatocytes. These tissue changes in testes might be due to the chronic inflammatory responses (Jones and Orosz, 2000; Nardoni et al., 2006; Femenia et al., 2007; Hussain et al., 2014); Solcan et al., 2018). Branched fungal hyphae and sporangium cultured from different tissues of ostrich observed in our study have also been reported (Khosravi et al., 2008).

\section{Conclusion}

In conclusion blocked bladder with penile protrusion and various pathological lesions observed in present study are valuable findings that would be helpful to avian physicians, wild life scientist to diagnose and treatment intervention in ostrich medicine practice.

Disclaimer: None.

Conflict of Interest: None. Source of Funding: None.

\section{References}

Adil M, Kandhare AD, Visnagri A, Bodhankar SL, 2015. Naringin ameliorates sodium arseniteinduced renal and hepatic toxicity in rats: decisive role of KIM-1, Caspase-3, TGF- $\beta$, and TNF- $\alpha$. Renal Fail. 37:1396-407.

Akkot A, Yilmaz R, Cangiil IT and Ozyigit MO, 2009. Pulmoneraspergillosis and amyloid accumulation in an ostrich. Turk. J. Vet. Anim. Sci. 33: 157-160.

Asan A, 2004.Aspeigillus, pemcillium, and related species reported from Turkey. Mycotaxon. 89: 155-157.

Atasever A and Gümüşsoy KS, 2004. Pathological, clinical and mycological findings in experimental aspergillosis infections of starlings. J. Vet. Med. A. 51:19-22.

Beckman BJ, Howe CW, Trampel DW, DeBey MC, Richard JL and Niyo Y, 1994. Aspergillus fumigatus keratitis with intraocular invasion in 15day-old chicks. Avian Dis. 38:660-665.

Beernaert LA, Pasmans F, Van Waeyenberghe L, Haesebrouck F and Martel A, 2010. Aspergillus infections in birds: a review. Avian Pathol. 39: 325-331.

Bezuidenhout AJ, 1999. Anatomy, In: The Ostrich Biology, Production and Health, Deeming, D.C. (ed.) CABI Publishing, Wallingford, UK, pp. 13-49. Cacciuttolo E, Rossi G, Nardoni S, Legrottaglie R and Mani P, 2009. Anatomopathological aspects of 
avian aspergillosis. Vet. Res. Comm. 33:521-527.

Cooper RG, 2005. Bacterial, Fungal and parasitic infections in the ostrich \{Stnrthiocamelusvar. domesticus). Anim. Sci. J. 76:97-106.

Cray C, Watson T, Rodriguez M and Arheart KL, 2009b.Application of galactomannan analysis and protein electrophoresis in the diagnosis of aspergillosis in avian species. J. Zoo Wildlife Med. 40:64-70.

Dawson TJ, Herd RM and Skadhauge E, 1985. Osmotic and ionic regulation during dehydration in a large bird, the emu (Dromaius novaehollandiae): An important role for the cloaca-rectum. Quart. J. Exper. Physiol. 70: 423 436.

Femenia F, Fontaine J, Lair-Fulleringer S, Berkova N, Huet D and Towanou N, 2007. Clinical, mycological and pathological findings in turkeys experimentally infected by Aspergillus fumigatus. Avian Pathol. 36-213-219.

Gulbahar MY, Agaoglu Z, Bivik H and Yuksek N, 2000.Zygomotic pro-ventriculitis and ventriculitis in ostriches (Struthio camelus) with impaction. Aust. Vet. J. 78: 247-249.

Hoppes S, Gurfield N, Flammer K, Colitz C and Fisher $\mathrm{P}, 2000$. Mycotic keratitis in a blue-fronted Amazon parrot (Amazona aestiva). J. Avian Med. Surg. 14: 185189.

Hussain R, Ali F, Rafique A, Ghaffar A, Jabeen G, Rafay M, Liaqat S, Khan I, Malik R, Khan MK, Niaz M, Akram K and Masood A, 2019. Exposure to sub-acute concentrations of glyphosate induce clinico-hematological, serum biochemical and genotoxic damage in adult cockerels. Pak. Vet. J. 39: $181-186$

Hussain R, Khan A, Mahmood F, Rehan S and Ali F, 2014. Clinico-hematological and tissue changes induced by butachlor in male Japanese quail (Coturnix japonica). Pest Biochem. Physiol. 109:58-63.

Jones MP and Orosz SE, 2000.The diagnosis of aspergillosis in birds. Seminars Avian Exotic Pet Med. 9:52-58.

Joseph V, 2000. Aspergillosis in raptors. Seminars Avian Exotic Pet Med. 9: 66-74.
Jung K, Kim Y, Lee H and Kim JT, 2009. Aspergillus fumigates infection in two wild Eurasian black vultures (Aegypiusmonachus linnaeus) with carbofuran insecticide poisoning: a case report. The Vet. J. 179: 307-312.

Khosravi AR, Shokri H, Ziglari T, Naeini AR, Mousavi Z and Hashemi H, 2008. Outbreak of severe disseminated aspergillosis in a flock of ostrich (Struthio camelus). Mycoses. 51:557-559.

Nardoni S, Ceccherelli R, Rossi G and Mancianti F, 2006. Aspergillosis in Larus cachinnans micaellis: survey of eight cases. Mycopathologia. 161:317-321.

Reissig EC, Uzal FA, Schettino A and Robles CA, 2002. Pulmonary aspergillosis in a great rhea (Rhea americana). Avian Dis. 46:754-756.

Sales J, 2006. Digestive Physiology and Nutrition of Ratites. Avian Poult. Biol. Rev. 17:41 -55

Shahrzad A, Ghalebi SR, Kheirandish R, Ghasemi N and Mohajeri FA, 2014. Aspergillosis and proventricular impaction in an ostrich (Struthio camelus). J. Coast. Life Med. 2: 662-664.

Viljoen M, Brand TS and Van der Walt JG, 2005. The effect of different dietary energy and protein levels on the digestive anatomy of ostriches. Proc. of the 3rd International Ratite Science Symposium and XII World Ostrich Congress, Madrid, 14 16th October 2005. p. 259.

\section{Contribution of Authors}

Hussain R: Conducted the study \& wrote manuscript.

Mahmood F: Conducted the study

Murtaza G: Involved in interpretation of results and manuscript preparation.

Abbas MI: Involved in interpretation of results and manuscript preparation.

Warraich JM: Involved in interpretation of results and manuscript preparation.

Akram R: Involved in interpretation of results and manuscript preparation. 